\title{
Qingkailing Injection Alleviates Experimental Autoimmune Uveitis in Rats via Inhibiting Th1 and Th17 Effector Cells
}

\author{
Qingmei Tian, ${ }^{a}$ Hongsheng Bi, ${ }^{* a, b, c}$ Yan Cui, ${ }^{a}$ Dadong Guo,${ }^{b, c}$ Xiaofeng Xie,${ }^{a}$ Weihua Su, ${ }^{a}$ and \\ Xingrong Wang ${ }^{a}$ \\ ${ }^{a}$ Affiliated Eye Hospital, Shandong University of Traditional Chinese Medicine; ${ }^{b}$ Eye Institute, Shandong University \\ of Traditional Chinese Medicine; and ${ }^{c}$ Key Laboratory of Integrated Traditional Chinese and Western Medicine for \\ Prevention and Therapy of Ocular Diseases, Universities of Shandong; No. 48, Yingxiongshan Road, Jinan City, \\ Shandong 250002, China. Received May 16, 2012; accepted August 9, 2012
}

\begin{abstract}
Qingkailing injection is a well-known composite formula of traditional Chinese medicine and is commonly used in clinical practice, which could offer immunomodulatory effect from our clinical experience on uveitis treatment by Qingkailing. We did the experiment in order to investigate the curative effect and mechanism of Qingkailing injection to rat experimental autoimmune uveitis (EAU). EAU was induced in Lewis rats by immunization IRBP1177-1191 in complete Freund's adjuvant in multi-point. We found that Qingkailing injection can alleviate autoimmune uveitis in rats, inhibit the differentiation toward Th1 and Th17 effector cells and the relevant cytokines secretion. The therapeutic effect may also be regulated through increased secretion of interleukin (IL)-10.
\end{abstract}

Key words Qingkailing injection; experimental autoimmune uveitis; Th1 cell; Th17 cell; HPLC; evaporative light scattering detector

Uveitis is a serious inflammation disease which is an important reason caused visual impairment mostly in young adults and children. ${ }^{1)}$ It is reported that about $10 \%$ of visual impairment are related with uveitis in America and accounts for the third reason of non-infectious blindness. ${ }^{2,3)}$

Autoimmune is considered as the key factor for the occurrence of uveitis. The normal tissue of eye contains a variety of antigens that could induce uveitis, such as retinal Santigen, interphotoreceptor retinoid-binding protein (IRBP), melanin associated antigen and so on. While the body's immune function is in disorder, the body may response to these proteins and results in the uveitis. Currently, corticosteroids and immunosuppressants are the main treatment approaches. Actually, they can strive to quickly suppress inflammation and reduce tissue damage, but serious side effects and immune suppression complications limit the usage of these treatment. Besides, it can lead to the uveitis chronic and recurrent. We use Qingkailing injection for uveitis patients combined with. By this way, steroids dose could be lower and it is easy to reduce its dosage.

Qingkailing injection is a well-known composite formula of traditional Chinese medicine and is commonly used in clinical practice. ${ }^{4,5)}$ It mainly comprises eight medicinal materials or extracts. It has been reported that the compounds of adenosine, geniposide, chlorogenic acid, baicalin, ursodeoxycholic acid, cholic acid and hyodeoxycholic acid are the major bioactive components which could synergistically contribute to its therapeutic effects. Moreover, these compounds are commonly used as markers for six important medicinal materials of Qingkailing injection. Currently, the efficacy, safety and batch-to-batch uniformity of Qingkailing injection were determined. ${ }^{6}$ Pharmacological studies proved that the Qingkailing can improve microcirculation, bring down a fever, anti-viral and antibacterial and it also can inhibit viral replication and inhibit the growth of multi-species of pathogenic bacteria. From our clinical experience on uveitis

The authors declare no conflict of interest. treatment by Qingkailing, we found Qingkailing could exert immunomodulatory effect and adjust the immune function of the body, so as to restore the immune balance.

In this study, we set up experimental autoimmune uveitis (EAU) model in Lewis rats through immunization by IRBP in Freund's Complete Adjuvant. Clinical manifestations and histopathological changes of EAU were used for efficacy evaluation of Qingkailing injection comparing with Dexamethasone. Furthermore, the effects of Qingkailing injection on uveitogenic effector Th1 and Th17 cells were evaluated by intracellular staining, enzyme-linked immunosorbent assay (ELISA) and optimized quantitative real-time polymerase chain reaction (Q-PCR) techniques.

\section{MATERIALS AND METHODS}

Animals and Reagents Female Lewis rats (160-180g) of specific pathogen-free grade were purchased from Peking Vital River Laboratory Animal Ltd., Beijing, China. All rats were fed and maintained in specific pathogen-free conditions according to the guidelines of Care and Use of Laboratory Animals published by the China National Institute of Health. All experimental procedures adhered to the Association for Research in Vision and Ophthalmology Statement for the use of animals in ophthalmic and vision research. Qingkailing injection and Dexamethasone were purchased from Hebei SHINEWAY Pharmaceutical Co., Ltd., China. Peptide R16 of bovine IRBP (residues 1177-1191, sequence ADGSSWEGVGVVPDV) and primers for PCR were synthesized by Shanghai Sangon Biological Engineering Technology \& Services Co., Ltd., China. Freund's Complete Adjuvant were purchased from Sigma-Aldrich, St. Louis, MO, U.S.A. Intracellular staining kit and ELISA kits, rat interleukin (IL)-2, fluorescein isothiocyanate (FITC) or phycoerythrin (PE) labeled antibodies for CD4, IL-17 and interferon (IFN)- $\gamma$ were purchased from eBioscience, San Diego, CA, U.S.A. Q-PCR kits were purchased from Aidlab Biotechnologies Co., Ltd., China. Analytical standards of adenosine, geniposide, 
chlorogenic acid, baicalin, ursodeoxycholic acid, cholic acid, and hyodeoxycholic acid were purchased from National Institute for the Control of Pharmaceutical and Biological Products (Beijing, China).

Chromatograms of Samples of Qingkailing Injection To detect the chromatograms of samples of Qingkailing injection by Alltech ELSD 2000 detector (Alltech, U.S.A.). The mobile phases were composed of water-acetic acid (100:0.5, A) and methanol (B). The gradient was as follows: $0 \mathrm{~min}, 95 \%$ A; $15 \mathrm{~min}, 88 \% \mathrm{~A}$; and $50 \mathrm{~min}, 25 \% \mathrm{~A} ; 75 \mathrm{~min}, 10 \% \mathrm{~A}$. Elution was performed at a solvent flow rate of $1.0 \mathrm{~mL} / \mathrm{min}$. The column compartment was kept at the temperature of $25^{\circ} \mathrm{C}$. The carrier gas was nitrogen $(99.999 \%)$, the drift tube temperature was set at $115^{\circ} \mathrm{C}$, and the gas flow rate was $3.2 \mathrm{~L} / \mathrm{min}$. The injection is $10 \mu \mathrm{L}$ loop.

Induction of Experimental Autoimmune Uveitis The peptide was prepared by emulsification of $100 \mu \mathrm{g}$ IRBP1177-1191 peptide in Freund's complete adjuvant containing $2.5 \mathrm{mg} / \mathrm{mL}$ of mycobacterium tuberculosis H37Ra in a total volume of $0.1 \mathrm{~mL}$. Rats were immunized by an injection of $0.1 \mathrm{~mL}$ peptide antigen in one footpad. The immunized rats were divided into three groups with 15 in each group. They were intervened from one day after immunization once every $2 \mathrm{~d}$. Control group were injected $2 \mathrm{~mL}$ of saline solution intraperitoneal. QKL group were injected intraperitoneal Qingkailing injection at a dose of $40 \mathrm{mg} / \mathrm{kg}$. Dex group were injected Dexamethasone at a dose of $0.25 \mathrm{mg} / \mathrm{kg}$. QKL group and Dex group were both diluted to $2 \mathrm{~mL}$ with normal saline.

Clinical and Histopathological Assessment All animals were monitored by slit-lamp biomicroscopy daily and scored according to acknowledged criteria. ${ }^{7)}$ The degree of eye inflammation was also confirmed by histopathological analysis on day 9. Briefly, the entire eye ball of euthanized animals was freshly dissected and fixed in $4 \%$ formaldehyde for $24 \mathrm{~h}$, before the corneas were cut and lens carefully removed. Fixed eyes were routinely embedded in paraffin blocks, and $2-\mu \mathrm{m}$ sections were stained with hematoxylin and eosin. The presence of disease was evaluated blindly by examining six sections cut through the pupillary-optic nerve plane at different levels for each eye. Severity of EAU was scored on a scale of 0 (no disease) to 4 (maximum disease). ${ }^{7)}$ QKL group were injected intraperitoneal Qingkailing injection at a dose of $40 \mathrm{mg} / \mathrm{kg}$. Dex group were injected Dexamethasone at a dose of $0.25 \mathrm{mg} / \mathrm{kg}$.

Preparation of IRBP1177-1191-Specific T Cells Briefly, 3 rats in each group were sacrificed on day 9 after immunization. $\mathrm{T}$ cells were isolated from lymph node cells and spleen cells by passage through a nylon wool column on day 9 after immunization, then $1 \times 10^{7}$ cells in $1 \mathrm{~mL}$ of RPMI 1640 medium in a 6 -well plate were stimulated for $48 \mathrm{~h}$ with $10 \mathrm{~g} /$ $\mathrm{mL}$ IRBP1177-1191 in the presence of $1 \times 10^{7}$ irradiated syngeneic spleen cells as antigen presenting cells in the presence of rat IL-2 $(10 \mathrm{ng} / \mathrm{mL})$, subsequently activated $\mathrm{T}$ cell blasts were separated by Ficoll gradient centrifugation, and cultured for another $72 \mathrm{~h}$. QKL group were injected intraperitoneal Qingkailing injection at a dose of $40 \mathrm{mg} / \mathrm{kg}$. Dex group were injected Dexamethasone at a dose of $0.25 \mathrm{mg} / \mathrm{kg}$.

Flow Cytometric Analysis of Surface and Intracellular Molecules For intracellular staining, IRBP1177-1191-specific $\mathrm{T}$ cells were stimulated with $20 \mu \mathrm{g} / \mathrm{mL}$ cell stimulation cocktail (which is a part of intracellular staining kit) for $5 \mathrm{~h}$. The stimulated cells were then stained for surface $\mathrm{CD}^{+}$(FITC and PE) and cultured for $45 \mathrm{~min}$. After washing and fixing, the cells were permeabilized overnight with fixation/permeabilization solution, then intracellular staining with antibodies against IFN- $\gamma$ (PE) and IL-17 (FITC) was performed for $45 \mathrm{~min}$. Finally, the treated cells were analyzed with Accuri C6 Flow cytometer. QKL group were injected intraperitoneal Qingkailing injection at a dose of $40 \mathrm{mg} / \mathrm{kg}$. Dex group were injected Dexamethasone at a dose of $0.25 \mathrm{mg} / \mathrm{kg}$.

ELISA To analyze the secretion of cytokines, $100 \mu \mathrm{L}$ supernatant was collected from the cultures of the IRBP1177-1191-specific T cells of each group on day 4 after culturing. Levels of interferon-gama (INF- $\gamma$ ), interleukin-17 (IL-17) and interleukin-10 (IL-10) were measured using commercially available ELISA kits according to the manufacturer's instructions. QKL group were injected intraperitoneal Qingkailing injection at a dose of $40 \mathrm{mg} / \mathrm{kg}$. Dex group were injected Dexamethasone at a dose of $0.25 \mathrm{mg} / \mathrm{kg}$.

Q-PCR To determine the level alteration of IFN- $\gamma$, IL-17 and IL-10 in rats after immunization, the Q-PCR was performed on day 3, day 5, day 7 and day 9. One spleen sample from normal rats without immunization was used as blank control. Briefly, freshly isolated spleens from each group were rapidly stored at $-80^{\circ} \mathrm{C}$. Total RNA was extracted from these tissues. First-strand cDNA was synthesized using $1 \mu \mathrm{g}$ of total RNA. The Q-PCR reaction was in a $20 \mu \mathrm{L}$ volume using $2 \times$ SYBR Green qPCR Mix. The PCR reaction was done using a real-time PCR System (Agilent Mx3000p, America) with an initial denaturation of $94^{\circ} \mathrm{C}$ for $10 \mathrm{~min}$, followed by 40 cycles of $94^{\circ} \mathrm{C}$ for $10 \mathrm{~s}, 55^{\circ} \mathrm{C}$ for $30 \mathrm{~s}$ and $72^{\circ} \mathrm{C}$ for $30 \mathrm{~s}$. Relative gene expression levels were quantified using the comparative $\Delta \mathrm{CT}$ method. Melting curve analyses and electrophoresis were performed to verify the specificity of the PCR products. The $\Delta \mathrm{CT}$ values were calculated as fold change in expression over spleen cells in $\mathrm{CM} \pm \mathrm{S}$.E. after normalization to respective endogenous $\beta$-actin control. QKL group were injected intraperitoneal Qingkailing injection at a dose of $40 \mathrm{mg} / \mathrm{kg}$. Dex group were injected Dexamethasone at a dose of $0.25 \mathrm{mg} / \mathrm{kg}$.

Statistical Analysis All the data were processed with SPSS 17.0. Each experiment was carried out in duplicate and repeated three times. Data were represented as the mean \pm standard deviation. All the data were processed for one-way analysis of variance (ANOVA) followed by post hoc analysis for significance with the LSD- $t$ multiple comparison test. $p<0.05$ was regarded as statistically significant.

\section{RESULTS}

Analysis of Qingkailing Injection To characterize Qingkailing injection, an HPLC/evaporative light scattering detector (ELSD) technique was applied. The chromatograms were shown in Fig. 1. As it can be seen, seven main ingredients (i.e., adenosine, geniposide, chlorogenic acid, baicalin, ursodeoxycholic acid, cholic acid and hyodeoxycholic acid) were found in Fig. 1B and, it is consistent with reference chemicals (Fig. 1A).

Qingkailing Injection Can Control the Experimental Autoimmune Uveitis in Rats The eyes in each group were observed everyday after immunization until day 17 post immunization, and clinical scores were recorded (Fig. 2). The results indicated that QKL group and Dex group developed 

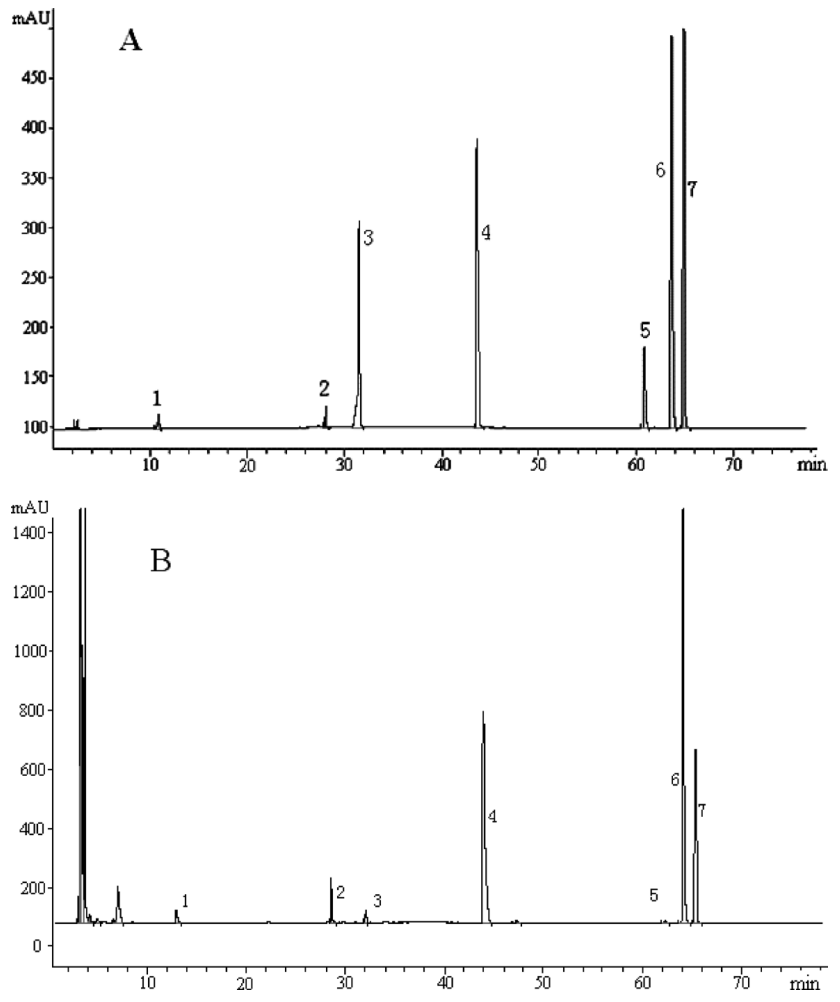

Fig. 1. Chromatograms of Qingkailing Injection and Reference Chemicals

The reference chemicals (A) and Qingkailing injection (B) were analyzed by HPLC/ELSD. 1: adenosine; 2: chlorogenic; 3: aeniposide; 4: baicalin; 5: ursodeoxycholic acid; 6: hyodeoxycholic acid; 7: cholic acid.

EAU later and with lighter inflammation than Control group. The clinical signs of Control group such as dilated or engorged blood vessels in iris were observed on day 8-9 post immunization. The most severe intraocular inflammation was detected on day 11-12 post immunization, as evidenced by an opaque anterior chamber and obscured pupil. On day 15 post immunization, the ocular inflammation was greatly resolved, with only minor clinical signs, and no inflammatory signs were detected on day 17 post immunization. Compared with Control group, in QKL group and Dex group, there were no inflammatory response on day 9 post immunization and only mild hyperemia of the iris, no hypopyon, normal pupil were observed on day 11 post immunization. On day 13 post immunization, the ocular inflammation was almost resolved, with only minor clinical signs. No inflammatory signs were detected on day 15 post immunization. Consistent with the results of the clinical scores, the histopathological performance also demonstrated retinal architecture was normal with only mild inflammatory cell infiltration in the eyes of the rats which accepted Qingkailing injection and Dexamethasone comparing with that of Control group (data not shown).

Differentiation of Th1 and Th17 Effector Cells Are Inhibited in the Action of Qingkailing Injection After treatment with Qingkailing injection and Dexamethasone in vitro, the levels of IFN- $\gamma$ were $15.4 \pm 4.12$ and $5.2 \pm 1.34 \%$, respectively. Whereas the level in control group was 71.3土 $5.72 \%$. Meanwhile, the levels of IL-17 were $8.5 \pm 3.35$ and $1.2 \pm 0.93 \%$, respectively. whereas the level in control group was $54.9 \pm 8.14 \%$. The expression levels of IFN- $\gamma$ and IL-17 in QKL group and DEX group both had a significant difference
(A)

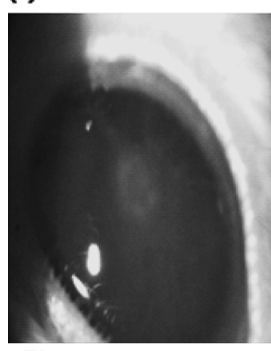

(B)

(C)

(D)

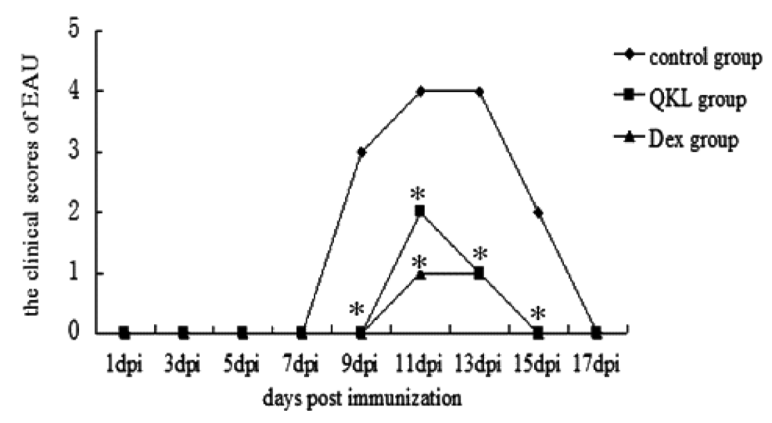

Fig. 2. Clinical Scores of the Monophasic EAU

Disease severity was observed daily by slit-lamp microscopy and graded as described in Materials and Methods. Based on the clinical course, monophasic EAU was divided into three stages: an initiation phase from the day of immunization to $8 \mathrm{~d}$ post immunization, an effector phase beginning from 8 to $15 \mathrm{~d}$ post immunization, with the peak inflammation obtained at $11 \mathrm{~d}$ post immunization, and a phase of resolution starting from $15 \mathrm{~d}$ post immunization. The rats in QKL group and Dex group developed EAU later and with lighter inflammation than in Control group (D). The eyes inflammation of Control group such as opaque anterior chamber and obscured pupil, red reflex absent and hyphema (A). QKL group such as slight iris angiectasis (B). Dex group were similar to QKL group and the iris angiectasis were more slight (C). Data are represented as the mean \pm standard deviation. The data were processed for one-way ANOVA and the difference had statistical significance $(n=3$ per group, $* p<0.05)$. dpi=day(s) post immunization. QKL group were injected intraperitoneal Qingkailing injection at a dose of $40 \mathrm{mg} / \mathrm{kg}$. Dex group were injected Dexamethasone at a dose of $0.25 \mathrm{mg} / \mathrm{kg}$.

compared to the relevant levels in Control group $(p<0.05)$. These results showed that the percentage of IFN- $\gamma$-expressing $\mathrm{CD}^{+}\left(\mathrm{CD}^{+} \mathrm{IFN}^{-} \gamma^{+}\right) \mathrm{T}$ cells and IL-17-expressing $\mathrm{CD}^{+}$ $\left(\mathrm{CD} 4^{+} \mathrm{IL}-17^{+}\right) \mathrm{T}$ cells differentiated from stimulated $\mathrm{T}$ cells were lower after treatment with Qingkailing injection and Dexamethasone in vitro (Figs. 3A,B), and this is consistent with the ELISA results. It revealed that the levels of IFN- $\gamma$ and IL-17 in the IRBP1177-1191-specific T cells supernatant of groups intervened by Qingkailing injection and Dexamethasone injection were markedly reduced compared with the control group (Fig. 4).

Th1 and Th17 Related Cytokine mRNA Expressions Were Inhibited by Qingkailing Injection IFN- $\gamma$ and IL-17 levels in rat spleen after immunization were determined by Q-PCR kinetically on day 3, 5, 7 and 9 post immunization. The relative expression of the normal rat was set as 1.00. Relative gene expression levels were quantified using the comparative $\Delta \mathrm{CT}$ value. We found that the mRNA expression levels of IFN- $\gamma$ and IL-17 in Control group increased gradually. Expression of IFN- $\gamma$ increased from day 3 and peaked on day 9 post immunization during the observation. Whereas, IL-17 expression increased on day 5 and peaked on day 9 post immunization (Figs. 5A, B). These quantitative PCR data also showed that Th1 and Th17 cells were at least two subsets of the major pathogenic effector $\mathrm{T}$ cells involved in severe ocular inflammation. Furthermore, traditional PCR technology was 
A

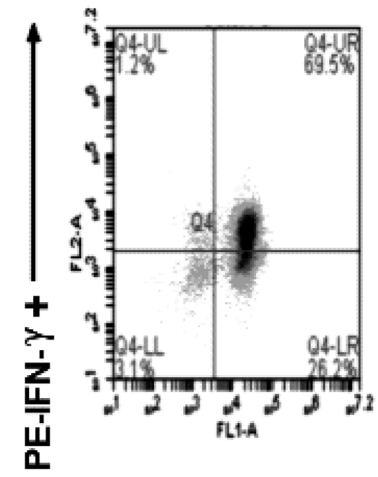

FITC-CD4+
QKL group

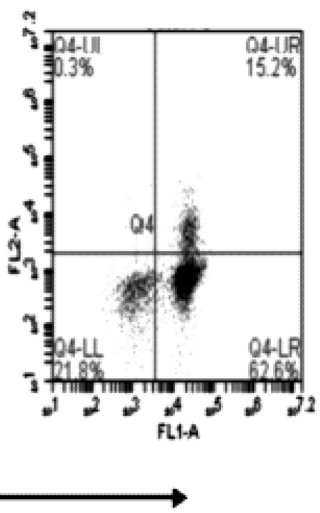

Dex group

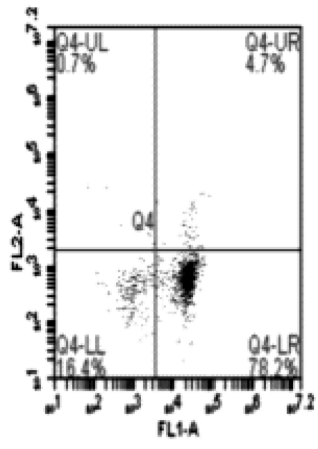

$\mathbf{B}$
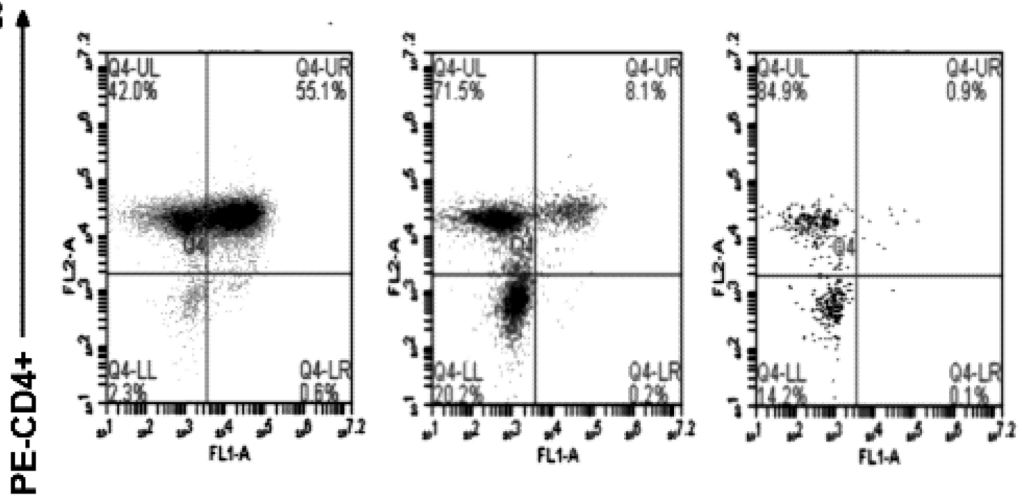

FITC-IL-17+

Fig. 3. Flow Cytometric Analysis of IL-17 and INF- $\gamma$ at Day 9

The result of the flow cytometric showed that the percentage of IFN- $\gamma$-expressing CD4 ${ }^{+} \mathrm{T}$ cells (A) and IL-17-expressing CD4 $4^{+} \mathrm{T}$ cells (B) are lower in vitro stimulated $\mathrm{T}$ cells of being intervened by Qingkailing injection or Dexamethasone injection compared to control group what immunized with IRBP1177-1191. Results of the percentage are expressed as mean \pm S.D. The data were processed for one-way ANOVA and the difference had statistical significance ( $n=3$ per group). QKL group were injected intraperitoneal Qingkailing injection at a dose of $40 \mathrm{mg} / \mathrm{kg}$. Dex group were injected Dexamethasone at a dose of $0.25 \mathrm{mg} / \mathrm{kg}$.

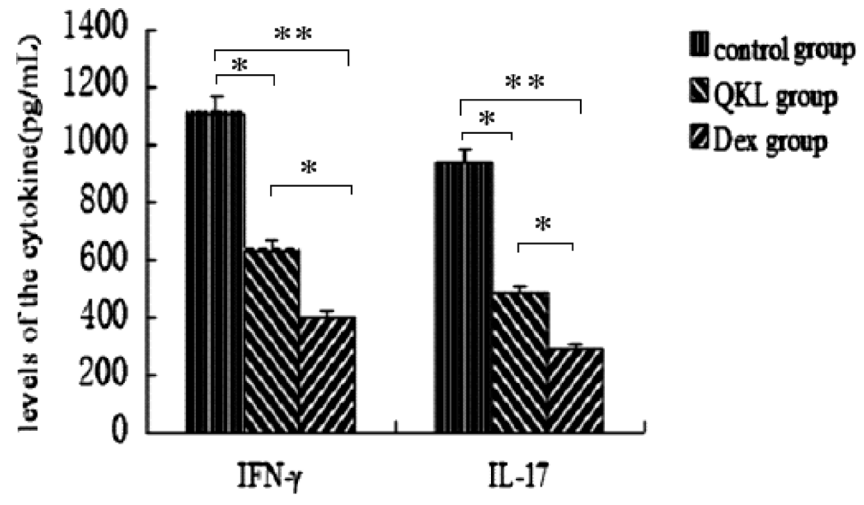

Fig. 4. The Expression Levels of IFN- $\gamma$ and IL-17 Determined by ELISA at Day 9

The expression levels of IFN- $\gamma$ and IL-17 were determined by ELISA. The levels of IFN- $\gamma$ and IL-17 in the supernatant of the IRBP1177-1191-specific T cells on the day 4 after cultured that were intervened by Qingkailing injection or Dexamethasone injection were markedly reduced compared to the control group immunized with IRBP1177-1191. The data were analyzed using one-way ANOVA. The difference among control group, QKL group and Dex group had statistical significance ( $n=3$ per group, $* p<0.05$ and $* * p<0.01)$. QKL group were injected intraperitoneal Qingkailing injection at a dose of $40 \mathrm{mg} / \mathrm{kg}$. Dex group were injected Dexamethasone at a dose of $0.25 \mathrm{mg} / \mathrm{kg}$.

also applied to verify some of the real-time PCR data. The transcripts from each sample were amplified from the cDNA using recombinant Taq polymerase (TaKaRa, Japan) under the following conditions: $94^{\circ} \mathrm{C}$ for $5 \mathrm{~min}, 94^{\circ} \mathrm{C}$ for $40 \mathrm{~s}, 58^{\circ} \mathrm{C}$ for $35 \mathrm{~s}$ and $72^{\circ} \mathrm{C}$ for $45 \mathrm{~s}$ for a total of 32 cycles. $\beta$-Actin was used as a loading control. The primers were as follows: $\beta$-actin: forward: 5 '-cacccgcgagtacaaccttc-3', reverse: $5^{\prime}$-cccataccaccatcacacc-3'; IFN- $\gamma$ : forward: 5'-ggatatctggaggaactggca-3', reverse: 5'-gctagattctggtgacagctg-3'. IL-17: forward: $5^{\prime}$-ttgctgctactgaacctggag-3', reverse: 5'-gcatggcggacaatagag-3'. Interestingly, we observed an important difference at the differentiation time points between Th1- and Th17-cells, that was Th1 differentiated earlier than Th17 in the immunized spleen (Figs. 5C-E). QKL group and Dex group, comparing with Control group, IFN- $\gamma$ and IL-17 mRNA levels decreased gradually (Figs. 5A, B). We have no further evidence to confirm the time of the decrease of IFN- $\gamma$ and IL-17 in Control group, but IL-17 mRNA expression lasts longer than IFN- $\gamma$ in C57BL/6 mice immunized by IRBP1-20 (paper had been submitted).

Qingkailing Injection Can Promote the Secretion of IL-10 We detected IL-10 levels of T cell supernatants from the three groups of rats by ELISA and we found the levels of IL-10 were markedly elevated in the action of Qingkailing injection and the Dexamethasone injection (Fig. 6A). Interestingly, to further characterize the function of Qingkailing injection, we found the expressions of IL-10 at the mRNA level were markedly elevated in the action of Qingkailing injection and the Dexamethasone injection (Fig. 6B). 


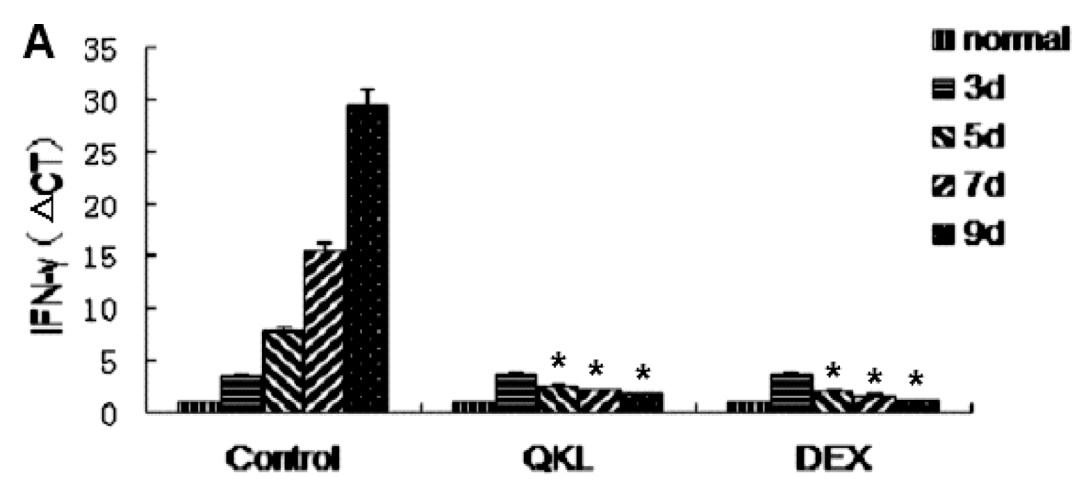

B
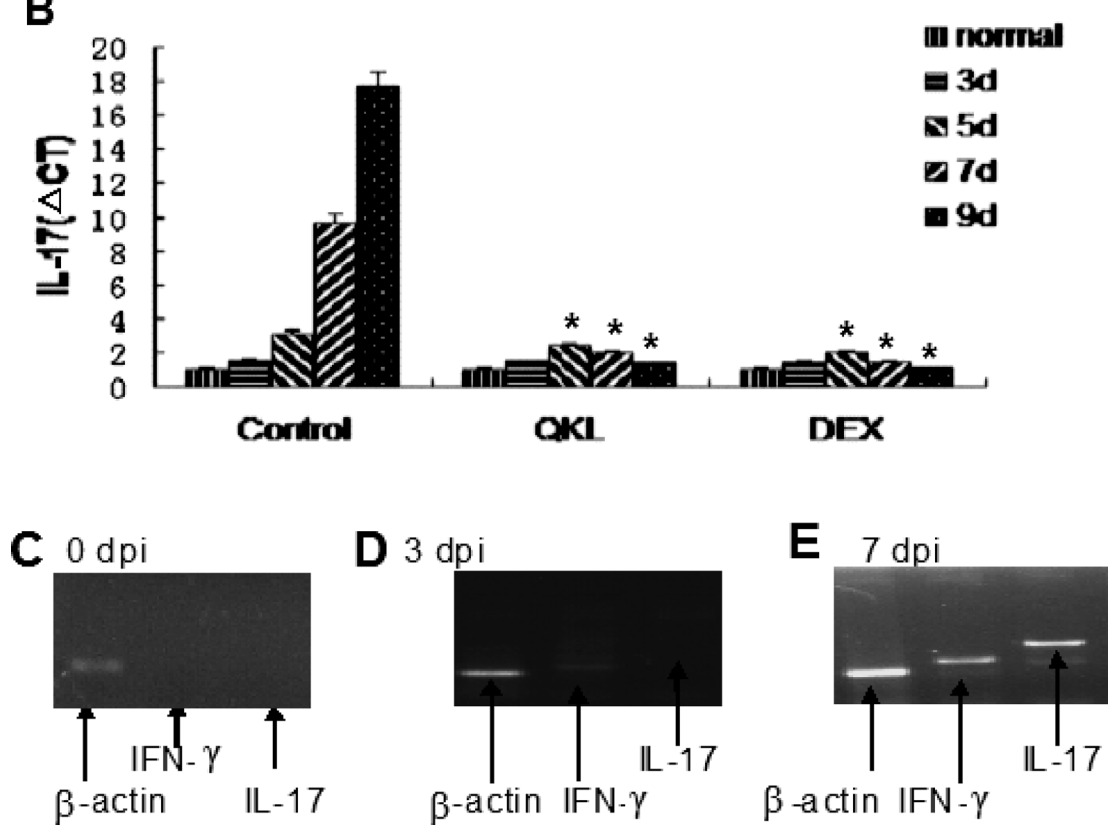

Fig. 5. The mRNA Levels of IFN- $\gamma$ and IL-17 after Different Treatment at a Definite Time

The mRNA levels of IFN- $\gamma$ and IL-17 were determined by Q-PCR (A, B) and traditional PCR technologies (C-E). The time series data are representative for experiments of immunization for inducing EAU model in Lewis rats in each group. The relative expression of the normal rat was set as 1.00. Relative gene expression levels were quantified using the comparative $\triangle \mathrm{CT}$ value. One-way ANOVA was to make statistics of every time series of each group and the difference had statistical significance at the same time point $(n=3, * p<0.05)$. The mRNA expression levels of IFN- $\gamma$ and IL-17 in control group increased gradually. Expression of IFN- $\gamma$ increased from day 3 and peaked on $9 \mathrm{~d}$ post immunization during the observation. Whereas, IL-17 expression increased on day 5 and peaked on $9 \mathrm{~d}$ post immunization. QKL group and Dex group, comparing with control group, IFN- $\gamma$ and IL-17 mRNA levels decreased gradually (A, B). Traditional PCR technology was applied to verify some of the realtime PCR data and confirmed that Th1 differentiated earlier than Th17 in the immunized spleen (C-E). dpi=day post immunization. QKL group were injected intraperitoneal Qingkailing injection at a dose of $40 \mathrm{mg} / \mathrm{kg}$. Dex group were injected Dexamethasone at a dose of $0.25 \mathrm{mg} / \mathrm{kg}$.

Additionally, we weighted the body weight prior to and after administration for each rat in our experiment. Because the dose of Qingkailing injection is very low when administered, little effect on the change of body weight in rats was observed.

\section{DISCUSSION}

Qingkailing injection is widely used in clinical practice. Pharmacological studies proved that Qingkailing have immunomodulatory effects and adjust the immune function of the body, so as to restore the normal state. In this study, we showed that Qingkailing plays an important role in inhibiting development of EAU in rats. We found that the rats which accepted Qingkailing injection did not develop EAU or had very lower clinical EAU scores. The inflammation occurred delayed or the course was shorter than in the control group. So we found Qingkailing injection can alleviate autoimmune uveitis in rats.

Previous studies indicate that Th1 cell play an important role in the attack of EAU. ${ }^{8)}$ Th1 cells could secrete IFN- $\gamma$. Recent studies have identified a unique subset of pathogenic $\mathrm{T}$ cells in autoimmune disease that expresses IL-17, but not IFN- $\gamma$ or IL-4. ${ }^{9-11)}$ And studies showed that this $\mathrm{IL}-17^{+}$autoreactive $\mathrm{T}$ cell subset plays a major role in the pathogenesis of autoimmune diseases. ${ }^{12-14}$ In the previous study, we reported that both $\mathrm{IL}-17^{+}$and IFN- $\gamma^{+}$IRBP-specific T cells are uveitogenic. ${ }^{15)}$ Meanwhile, we found that in QKL group and Dex group, the differentiation of IFN- $\gamma$-expressing $\mathrm{CD}^{+} \mathrm{T}$ cells and IL-17-expressing $\mathrm{CD}^{+}{ }^{+} \mathrm{T}$ cells are lower than control cells. We also observed that the relative expression of Th1- and Th17-cytokines at the mRNA level were markedly reduced after treatment with Qingkailing injection and Dexamethasone. Interestingly, we noted an important difference of the differentiation time points between Th1- and Th17-cells that Th1 differentiated earlier than Th17 in spleen. After treatment 
A

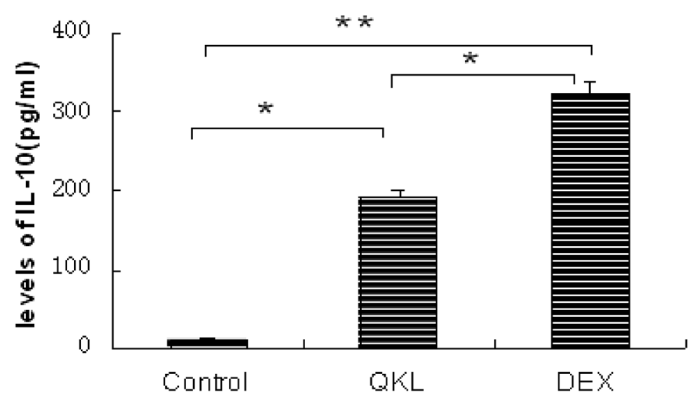

B

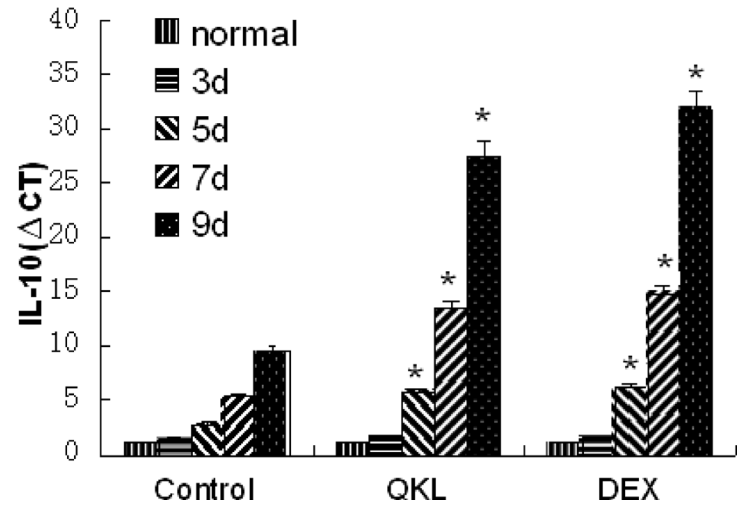

Fig. 6. Qingkailing Injection Promotes the Secretion of IL-10

IL-10 levels from T cell supernatants in control group, QKL group and Dex group were measured by ELISA. IL-10 levels in rat spleen after immunization were determined by Q-PCR kinetically on 3, 5, 7 and $9 \mathrm{~d}$ post immunization. The data were processed for one-way ANOVA. The difference between control group and QKL group or Dex group had statistical significance ( $n=3$ per group, ${ }^{*} p<0.05$ an ${ }^{* *} p<0.01$ ) and the difference between QKL group and Dex group also had statistical significance $(n=3$ per group, $* p<0.05)$. The levels of IL-10 were markedly elevated in the action of Qingkailing and Dexamethasone. QKL group were injected intraperitoneal Qingkailing injection at a dose of $40 \mathrm{mg} / \mathrm{kg}$. Dex group were injected Dexamethasone at a dose of $0.25 \mathrm{mg} / \mathrm{kg}$.

with Qingkailing injection and dexamethasone, the levels of IFN- $\gamma$ and IL-17 secreted from the IRBP1177-1191-specific $\mathrm{T}$ cells were markedly decreased comparing with the control. These indicated that the Qingkailing may have similar anti-inflammatory effect as Dexamethasone. They can suppress $\mathrm{T}$ cells differentiation to Th1 and Th17 and inhibit the expression of Th1 and Th17 signature cytokines.

Many studies indicted that the IL-10 had marginal roles in suppressing uveitis. ${ }^{16)}$ And in the study, we found the expression of IL-10 at the mRNA level were markedly elevated in the action of Qingkailing injection and Dexamethasone. The levels of IL-10 were significantly elevated in the supernatant of the IRBP1177-1191-specific T cells that were intervened by Qingkailing injection and Dexamethasone. These indicated that the Qingkailing can promote the secretion of IL-10.

In a summary, Qingkailing injection can alleviate autoimmune uveitis in rats. It mainly exerts anti-inflammatory effects by inhibiting the differentiation of the Th1 and Th17 effector cells and reducing secretion of the corresponding cytokines. This maybe involves in the regulation of cytokine IL-10 .

Acknowledgments This study was supported by the National Natural Science Foundation of China (81072961 and 81100658) and the National Science Foundation for
Post-doctoral Scientists of China (2012M510068). We greatly appreciate the Experimental Centre of Shandong University of Traditional Chinese Medicine for HPLC/ELSD analysis.

\section{REFERENCES}

1) Rothova A, Suttorp VS, Frits T, Kijlstra A. Causes and frequency of blindness in patients with intraocular inflammatory disease. $B r . J$. Ophthalmol., 80, 332-336 (1996).

2) Caspi RR. A look at autoimmunity and inflammation in the eye. $J$. Clin. Invest., 120, 3073-3083 (2010).

3) Nussenblatt RB. The natural history of uveitis. Int. Ophthalmol., 141, 303-308 (1990).

4) Qi ZJ, Qian JJ, Qiao TX, Hou WH. A preliminary biochemical study on the protective effects of Qingkailing injection on liver injury. J. Tradit. Chin. Med., 3, 27-31 (1983).

5) Hua Q, Zhu XL, Li PT, Tang HL, Cai DY, Xu Y, Jia X, Chen J, Shen Y. Refined Qing Kai Ling, traditional Chinese medicinal preparation, reduces ischemic stroke-induced infarct size and neurological deficits and increases expression of endothelial nitric oxide synthase. Biol. Pharm. Bull., 31, 633-637 (2008).

6) Yan SK, Xin WF, Luo GA, Wang YM, Cheng YY. Simultaneous determination of major bioactive components in Qingkailing injection by high-performance liquid chromatography with evaporative light scattering detection. Chem. Pharm. Bull., 53, 1392-1395 (2005).

7) Agarwal RK, Caspi RR. Rodent models of experimental autoimmune uveitis. Methods Mol. Med., 102, 395-419 (2004).

8) Caspi RR. Th1 and Th2 responses in pathogenesis and regulation of experimental autoimmune uveoretinitis. Int. Rev. Immunol., 21, 197-208 (2002).

9) Mangan PR, Harrington LE, O'Quinn DB, Helms WS, Bullard DC, Elson CO, Hatton RD, Wahl SM, Schoeb TR, Weaver CT. Transforming growth factor- $\beta$ induces development of the TH17 lineage. Nature, 441, 231-234 (2006).

10) Park $\mathrm{H}$, Li Z, Yang $\mathrm{XO}$, Chang SH, Nurieva R, Wang YH, Wang Y, Hood L, Zhu Z, Tian Q, Dong C. A distinct lineage of CD4 T cells regulates tissue inflammation by producing interleukin 17. Nat. Immunol., 6, 1133-1141 (2005).

11) Liang SC, Tan XY, Luxenberg DP, Karim R, Dunussi-Joannopoulos K, Collins M, Fouser LA. Interleukin (IL)-22 and IL-17 are coexpressed by Th17 cells and cooperatively enhance expression of antimicrobial peptides. J. Exp. Med., 203, 2271-2279 (2006).

12) Komiyama $Y$, Nakae $S$, Matsuki $T$, Nambu A, Ishigame $H$, Kakuta S, Sudo K, Iwakura Y. IL-17 plays an important role in the development of experimental autoimmune encephalomyelitis. J. Immunol., 177, 566-573 (2006).

13) Langrish CL, Chen Y, Blumenschein WM, Mattson J, Basham B, Sedgwick JD, McClanahan T, Kastelein RA, Cua DJ. IL-23 drives a pathogenic $\mathrm{T}$ cell population that induces autoimmune inflammation. J. Exp. Med., 201, 233-240 (2005).

14) Lubberts E, Joosten LA, Oppers B, van den Bersselaar L, Coenen-de Roo CJ, Kolls JK, Schwarzenberger P, van de Loo FA, van den Berg WB. IL-1-independent role of IL-17 in synovial inflammation and joint destruction during collagen-induced arthritis. $J$. Immunol., 167, 1004-1013 (2001).

15) Peng Y, Han G, Shao H, Wang Y, Kaplan HJ, Sun D. Characterization of IL-17 $7^{+}$interphotoreceptor retinoid-binding protein-specific T cells in experimental autoimmune uveitis. Invest. Ophthalmol. Vis. Sci., 48, 4153-4161 (2007).

16) Lee YS, Amadi-Obi A, Yu CR, Egwuagu CE. Retinal cells suppress intraocular inflammation (uveitis) through production of interleukin-27 and interleukin-10. Immunology, 132, 492-502 (2011). 